\title{
Effects of tritiated thymidine on human neural stem and progenitor cells
}

\author{
Christine Granotier-Beckers ${ }^{1, *}$, Sofiane Mokrani ${ }^{1}$, and François D. Boussin ${ }^{1}$ \\ ${ }^{1}$ CEA, JACOB, IRCM, INSERM U967, Laboratoire de Radiopathologie, Université Paris Diderot \\ Sorbonne Paris Cité, Université Paris XI, 92260 Fontenay-aux-Roses, France
}

Ionizing radiation during pregnancy may impair brain development resulting in microcephaly and mental retardation. These effects might be related to the targeting of highly proliferating NSPC during brain development. These cells give rise to the main types of neural differentiated cells of the brain.

Tritium which is the radioactive hydrogen isotope emitting $\beta$-particles is either of natural origin or is produced by nuclear facilities for fusion and fission reactions. Tritium dispersion around nuclear facilities may result in people exposure to very low doses of tritium in different chemical forms (tritium gas, tritiated water or organically bound tritium).

Today, low dose exposition to tritium and its consequences on human health are still poorly known, in particular for the developing brain. By contrast, previous studies have shown that prenatal exposition to high doses of tritiated water or tritiated thymidine induce microcephaly and neurobehavioral changes in rat and mice models [1-3].

We have thus investigated the effects of tritiated thymidine on primary cultures of human neural stem and progenitor cells from several donors. Tritiated thymidine is incorporated in DNA during replication leading to chronic irradiation of the contaminated cells and their progeny.

We have shown that tritiated thymidine incorporation led to dose-dependent decrease of cell proliferation in NSPC. This effect was due to inhibition of cell cycle progression and induction of apoptosis and premature senescence. Tritiated thymidine also impaired neural stem cells functions by triggering their premature differentiation.

Intracellular tritium dosimetry based on autoradiography revealed that cellular effects were only associated to high levels of tritiated thymidine incorporation.

We then compared the chromosome instability induced by tritiated thymidine after several rounds of replication. Cytogenetic analysis of metaphase spreads evidenced that tritiated thymidine induced chromatid-type aberrations after the first round of incorporation, consistently with the tritiated thymidine incorporation in the newly synthesized DNA strands. Tritium dosimetry revealed that one chromatid break was detected for about 50 tritium disintegrations occurring during $\mathrm{S}$ and $\mathrm{G} 2 / \mathrm{M}$ phases.

Both chromatid-type and chromosome-type aberrations are detected in $\gamma$-irradiated cycling cells, resulting from DNA damage induced before or after DNA replication respectively. Interestingly, chromatid-type aberrations were still mainly induced by tritiated

* Corresponding author: christine.granotier@cea.fr 
thymidine incorporation during several cell cycles whereas very few chromosome-type aberrations were observed. Moreover, the number of chromosome aberrations did not increase along the successive rounds of tritium incorporation, suggesting that contaminated cells developed an adaptive response. Further studies are required to investigate the molecular mechanisms involved.

\section{References}

1. S. Zamenhof, E. van Marthens, Radiat Res, 77 (1979)

2. SJ. Bursian, DF. Cahill, JW. Laskey, LN. Parker, Int J Radiat Biol Relat Stud Phys Chem Med, 27,5 (1975)

3. B. Wang, H. Takeda, WM. Gao, XY. Zhou, T. Odaka, H. Ohyama, Health Phys. 77, 1(1999). 